\title{
A Distributed Topology Control Technique for Low Interference and Energy Efficiency in Wireless Sensor Networks
}

\author{
Tapiwa M. Chiwewe, Student Member, IEEE, and Gerhard P. Hancke, Senior Member, IEEE
}

\begin{abstract}
Topology control plays an important role in the design of wireless ad hoc and sensor networks; it is capable of constructing networks that have desirable characteristics such as sparser connectivity, lower transmission power and a smaller node degree. In this research a new distributed topology control technique is presented that enhances energy efficiency and reduces radio interference in wireless sensor networks. Each node in the network makes local decisions about its transmission power and the culmination of these local decisions produces a network topology that preserves global connectivity. Central to this topology control technique is the novel Smart Boundary Yao Gabriel Graph (SBYaoGG) and optimizations to ensure that all links in the network are symmetric and energy efficient. Simulation results are presented demonstrating the effectiveness of this new technique as compared to other approaches to topology control.
\end{abstract}

Index Terms - Graph theory, power control, proximity graphs, topology control, wireless sensor networks.

\section{INTRODUCTION}

M ULTIFUNCTIONAL wireless sensor nodes are a development brought about by recent advancements in wireless communications and electronics [1]. These sensor nodes are small in size and communicate unrestrictedly over short distances. They have sensing, data processing and communication capabilities and their features have enabled, as well as provided, impetus to the idea of Wireless Sensor Networks (WSNs). WSNs are powerful in that they are amenable to support a lot of real world applications that vary considerably in terms of their requirements and characteristics.

Networks of sensors exist in many industrial applications providing the ability to monitor and control the environment in real time. Most of these networks however are wired and as a result are costly to install and maintain. To lower the system and infrastructure costs wireless solutions can be used [2]. Wireless solutions have other benefits in industrial applications such as enhanced physical mobility, reduced danger of breaking cables, less hassle with connectors and ease of upgrading [3].

WSNs can be deployed in unpleasant, inaccessible or hazardous environments which are impractical with traditional wired networks such as in the bearings of motors or the inside of whirring motors [4]. In addition to this, the collaborative nature of WSNs brings about flexibility, self-organization, self-configuration, inherent intelligent-processing capability and enables rapid deployment.

WSNs therefore, are an attractive option for industrial applications. They are beginning to substitute the conventional industrial wired communication systems [5] in applications such as factory automation, distributed control systems, environment and equipment monitoring, product tracking, automotive systems and other kinds of networked embedded systems. WSNs play a crucial part in creating a highly reliable and self-healing industrial system that is highly responsive to real-time events with appropriate actions [6]. The data collected by sensor nodes is sent wirelessly to a sink node that analyses the data from each sensor node. Notifications of any possible problems can then be sent to plant personnel or instructions can be relayed back to the senor nodes to activate on board actuators in a certain way if equipped.

The major technical challenges for realization of industrial WSNs are resource constraints [7], dynamic topologies and harsh environmental conditions, Quality-of-Service (QoS) requirements, data redundancy, packet errors and variablelink capacity, security, large-scale deployment and ad hoc architecture [8], and integration with internet and other networks [9]. Most QoS metrics are interdependent such that improving one may degrade the other. As a result of this system designers have to achieve best trade-offs between different QoS metrics [10].

More fundamental to the design of sensor networks, however, is that sensor nodes have a severely constrained energy allocation due to the limited power supply from batteries and as a result energy-efficiency is the most important figure of merit in WSNs [3]. Internal power sources help to eliminate the need for wires to the nodes and allow greater mobility. Part of the current vision for WSNs is to have sensor nodes that can last forever without external power sources or having to change their batteries. Energy harvesting techniques can be used to achieve this such as piezoelectric 
power generation in which a micro-electro-mechanical systems (MEMS) cantilever converts mechanical motions into electrical power. Two mechanisms that have been explored in order to propel the cantilever's movements are the use of a radioactive isotope and the harvesting of vibrations from the environment [11].

In a densely deployed wireless network, a single node will have many neighboring nodes which it can communicate with directly when using sufficiently high transmission power. However, high transmission power requires a lot of energy and using many neighbors is a burden for a medium access control (MAC) protocol. Routing protocols suffer volatility in the network when nodes move around and frequently sever or form many links between one another [12]. To provide nodes with a long period of autonomy, protocols that aim to optimize power usage are needed, so as to extend the lifetime of the nodes and the lifespan of the network as a whole [13].

In order to meet these challenges, topology control (TC) can be applied. Topology control is one of the most important techniques used in WSNs to reduce energy consumption and radio interference [14]. It lends itself to the mechanisms of multi-hop communication and energy-efficient operation. Topology control aims to control the graph representing communication links between nodes, with the purpose of meeting a global property of the graph - such as connectivity, while reducing energy consumption and radio interference.

\section{TOPOLOGY CONTROL}

\section{A. Taxonomy}

There are several different approaches to topology control and it is possible to organize them into a coherent taxonomy. The first distinction is between approaches that control transmitter power and those that impose a hierarchy in the network. Hierarchical approaches change the logical structure of the network in terms of node adjacencies and may be broken down into approaches that use clustering and those that use dominating sets.

The power control approaches act on the transmission power of nodes using several different techniques. The first distinction to make of power control approaches is between homogeneous and non-homogeneous approaches. Homogeneous topology control is the easier of the two in which nodes are assumed to use the same transmitting power and the problem of topology control becomes in essence one of finding the value of the transmitter range $r$ that satisfies a certain network wide property.

In non-homogenous topology control nodes are allowed to select different individual transmitting powers up to a certain maximum that they can support which means that they will have different transmitting ranges. This form of topology control can be split into three different categories according to the type of information that is used to generate the topology. These three categories are location based, direction based and neighbor based.

In location based approaches exact node locations are known and are either used by a centralized authority to calculate a set of transmitting range assignments which optimize a certain measure or are exchanged between nodes to create an approximately optimal topology in a distributed fashion. In direction based approaches nodes are assumed to not know their positions but can estimate the relative direction to each of their neighbors. Lastly in neighbor based approaches the only knowledge nodes have of their neighbors is the neighbor IDs and the IDs are ordered according to some criterion when performing topology control.

\section{B. Quality Measures}

Different approaches to topology control will produce different results. For a collection of nodes $V$, let $G$ denote the graph on $V$ for which there is an edge from node $u$ to node $v$ only if $u$ can directly reach $v$. It is desirable to judge the usefulness of a topology $T$ returned by a topology control algorithm and compare it with results from other algorithms. In order to do this, some metrics and measures are required which include connectivity, energy efficiency, throughput and robustness to mobility [15].

Connectivity - If there is a multi-hop path between $u$ and $v$ in $G$ then there should also be a path in $T$. This is a basic requirement for a topology control algorithm, that it should not disconnect a connected graph.

Energy efficiency - The energy consumed for a transmission between $u$ and $v$ is a polynomial function of the distance between $u$ and v. Two common notions of energy efficiency are the energy stretch factor and the hop stretch factor. The energy stretch factor is the worst increase in energy used to deliver a packet between any pair of nodes $u$ and $v$ along a minimum energy path between the original graph $G$ and the topology controlled graph $T$. The hop stretch is similar except that the focus is on path length as opposed to energy consumption. Formally,

$$
\text { energy stretch factor }=\max _{u, v \in V} \frac{E_{T}(u, v)}{E_{G}(u, v)}
$$

where $E_{G}(u, v)$ is the energy consumed along the most energy-efficient path in graph $G$. Likewise,

$$
\text { hop stretch factor }=\max _{u, v \in V} \frac{\left|(u, v)_{T}\right|}{\left|(u, v)_{G}\right|}
$$

where $(u, v)_{G}$ is the shortest path in graph $G$ and $\left|(u, v)_{G}\right|$ is its length.

Node degree - In order to better evaluate the performance of the topology control technique in terms of interference, a distinction is made between the physical and the logical node degree. The physical node degree refers to the number of neighbor nodes that are within the transmitter range of a given node. The logical node degree refers to the number of neighbor nodes that a given node is linked to. Simplicity and maintainability - It is desirable for a topology $T$ to be simple and easy to maintain and objective measures that can be used to evaluate these subjective goals are the number of edges in $T$ and the maximum node degree (number of neighbors) of any node in $T$. It is desirable also for the algorithm used to have little overhead in terms of computation and communication requirements. 
Throughput - It is desirable for the network topology $T$ to have a high throughput, where it is possible to sustain a comparable amount of traffic as the original network topology $G$. Several throughput measures can be used [15] one of which is the bit-meter which is defined in terms of the bit-distance product. A network transports one bit-meter when it one bit is transported a distance of one meter. The throughput of the network is then the number of bit-meters transported per second.

Robustness to mobility - The mobility of nodes causes neighborhood relationships to change in the original graph $G$ and some other nodes will have to change their topology information. A robust topology should only require a small number of these adaptations and avoid the effects of reorganization due to local node movement affecting the entire network. A measure of robustness is the adaptability [15] which is the maximum number of nodes that need to change their topology information as a result of the movement of a node. Adaptability depends on the size of the transmission neighborhood of the mobile node $u$ and the relative location of the nodes. Robustness to mobility is an issue in industrial applications such as product and material tracking.

\section{Power Control}

\section{1) Non Homogeneous}

\section{a) Range Assignment}

The range assignment problem $\left(\mathrm{R}_{\mathrm{A}}\right)$ is that of assigning transmitting ranges to individual nodes in the network such that the resulting communication graph $T$ is strongly connected and the energy cost is at a minimum. Let $N=$ $\left\{u_{1}, \ldots, u_{n}\right\}$ be a set of points in region $R=[0, l]^{d}$ for $d=$ $1,2,3$, denoting the positions of network nodes. A more formal definition of the range assignment problem is that of finding the connecting range assignment RA such that

$$
c(R A)=\sum_{u_{i} \in N}\left(R A\left(u_{i}\right)\right)^{\alpha}
$$

is at a minimum. The problem is solvable in polynomial time $\left(O\left(n^{4}\right)\right)$ in the case of one dimension while it is NP-hard in the cases of 2 and 3 dimensions. Computing the optimal range assignment in dimensions higher than the one dimensional case is therefore extremely difficult. It is possible however to approximate the optimal solution within a factor of 2 if for every node $u_{i} \in N, R A\left(u_{i}\right)$ is defined as the maximum of distances $\delta_{u_{i}, u_{j}}$ for all nodes $u_{j}$ that are neighbours of $u_{i}$ in $T$.

The communication graph generated by a range assignment is generally not symmetric as it may contain some unidirectional links. MAC protocols are designed to work under the assumption of symmetric links so it is not desirable to implement wireless unidirectional links given the inefficiency and overhead that will be added. Two variants of $\mathrm{R}_{\mathrm{A}}$ based on the concept of symmetry are the symmetric range assignment problem $\left(\mathrm{S}_{\mathrm{RA}}\right)$ and the weakly symmetric range assignment problem $\left(\mathrm{W}_{\mathrm{SRA}}\right)$.

For an arbitrary communication graph $G$, the symmetric sub-graph of $G$ denoted $G_{S}$ is obtained by deleting all unidirectional links which are edges such that $u, v \in E$ but $v, u \notin E . \mathrm{W}_{\mathrm{SRA}}$ is then solved by determining the range assignment RA such that the symmetric sub-graph $G_{S}$ is connected and $c(R A)=\sum_{u_{i} \in N}\left(R A\left(u_{i}\right)\right)^{\alpha}$ is at a minimum. $\mathrm{S}_{\mathrm{RA}}$ is solved by determining a symmetric range assignment that generates a communication graph that contains only bidirectional links where $R A\left(u_{i}\right) \geq \delta_{u_{i}, u_{j}} \Leftrightarrow R A\left(u_{j}\right) \geq \delta_{u_{i}, u_{j}}$ such that $c(R A)=\sum_{u_{i} \in N}\left(R A\left(u_{i}\right)\right)^{\alpha}$ is minimum. $\mathrm{S}_{\mathrm{RA}}$ is NPhard in the 2 and 3 dimensional cases and $W_{\text {SRA }}$ does not reduce the computational complexity of the problem.

\section{b) Minimum Energy Unicast}

In minimum energy unicast the goal is to compute topologies that have energy-efficient paths between potential source-destination pairs. For a connected communication graph $G$ obtained when all nodes transmit at maximum power, every edge $\left(u_{i}, u_{j}\right)$ is weighted with the power $\delta_{u_{i}, u_{j}}^{\alpha}$ necessary to transmit between $u_{i}$ and $u_{j}$. These edge weights are used to calculate the power stretch factor for a given path $P=u_{1}, u_{2}, \ldots, u_{k}$ in $G$. The goal can then be achieved by identifying a sub-graph $T$ of the maximum power graph $G$ that has a low power stretch factor and is sparser than the original graph. Graph $T$ can then be used to compute routes between nodes and has the advantage that computing routes in $T$ is easier than in $G$, it involves less message overhead and requires less maintenance in the presence of node mobility.

The routing sub-graph $T$ should ideally have the following features:

a) Constant power stretch factor i.e. $T$ should be a power spanner of $G$. This ensures that the routes calculated on $T$ are at most a constant factor away from the energy optimal routes.

b) Linear number of edges i.e. $T$ must be sparse. This eases the task of finding routes in $T$ and maintaining the routing graph in the presence of node mobility. It also reduces the routing overhead.

c) Node degree must be bounded. This is because nodes with a high degree have a high likelihood of being bottlenecks in the communication graph.

d) Easy computation in distributed and localized fashion where only information provided by neighbor nodes in $G$ is used. This is essential for fast and effective computation of the routing graph in a real WSN.

There are a number of geometric graphs that satisfy the above requirements and are based on sub-graphs of $G$. These include the Relative Neighborhood Graph (RNG) [16], the Gabriel Graph (GG) [12], the Delaunay Graph (DG) [14] and the Yao Graph (YG) [17]. These graphs are called proximity graphs since the set of neighbors for any node $u$ can be calculated using the position of neighbor nodes in the original graph $G$. Proximity graphs therefore satisfy property d) above. Different quality measures of these proximity graphs are compared in TABLE 1. 
TABLE 1

\begin{tabular}{|l|c|c|c|}
\multicolumn{2}{|c|}{ QUALITY MEASURES OF DIFFERENT PROXIMITY GRAPHS [14] } \\
& $\begin{array}{c}\text { Distance } \\
\text { Stretch Factor }\end{array}$ & Power Stretch Factor & Node Degree \\
\hline RNG & $n-1$ & $n-1$ & $n-1$ \\
\hline GG & $\frac{\sqrt{n-1}}{2} \pi$ & $\left(\frac{1+\sqrt{5}}{2} \pi\right)^{\alpha}$ & $\Theta(n)$ \\
\hline RDG & $\frac{1+\sqrt{5}}{1}$ & $\frac{1}{1-\left(2 \sin \frac{\pi}{e}\right)^{\alpha}}$ & $n-1$ \\
\hline YG & $\frac{1-2 \sin \frac{\pi}{e}}{}$ & & \\
\hline
\end{tabular}

\section{c) Protocols}

Different solutions to the problem of non-homogeneous topology control have been presented covering range assignment and energy-efficient unicast and broadcast. These solutions have sought to optimise a certain network measure with the emphasis being on the quality of the topology produced. A more practical approach to the topology control problem involves designing simple, fully distributed protocols that build and maintain a fairly good topology. These protocols are called topology control protocols and may be position based such as the Local Minimum Spanning Tree (LMST) [18], direction based such as Cone Based Topology Control (CBTC) [19] or neighbor based such as the $K$-NEIGH protocol [20].

\section{Mobile Networks}

Most of the topology control techniques highlighted are for stationary networks with some being adaptable to mobile networks. Mobility can occur in the case of the physiological monitoring of workers or the tracking of products or when sensors are attached to mobile equipment such as forklifts. Mobility in networks affects topology in a number of ways [14] notably increased message overhead and non-uniform node spatial distribution.

\section{SMART BOUNDARY YAO GABRIEL GRAPH}

\section{A. Objectives}

There were two design objectives in developing the envisaged topology control technique for WSNs. The first objective was that it should be energy efficient and the second was that it should have low interference. Performance measures were used to determine how well these objectives were met. The relative performance of the new technique compared to other well-known approaches to topology control was also used to evaluate how well these objectives were met.

These objectives, however are competing as improving energy efficiency, as measured through the energy stretch factor of a generated topology, increases the level of interference in the network as measured by the maximum and average node degree of the generated topology. In addition to this, topology control involves a compromise between energy conservation and network connectivity. The topology control technique that was developed was designed to meet the two competing objectives given above by finding a balance between them using certain heuristics given in section III.C.2) below.

\section{B. Requirements}

To meet the set out design objectives, the routing sub-graph $T$ produced by the topology control technique from the original graph $G$ had to meet certain requirements. A number of the requirements were for minimum energy unicast; these are:

1. Constant power stretch factor, i.e. the graph should be a power spanner of $\mathrm{G}$.

2. Linear number of edges, i.e. the graph must be sparse.

3. Easy computation in a distributed and localized way.

In addition to this, the sub-graph $T$ had to be:

1. Connected with high probability if the original graph $\mathrm{G}$ is connected.

2. Planar, meaning that no two edges in the graph cross each other. This will enable some localized routing algorithms to work with the generated topology such as Greedy Face Routing (GFR), Greedy Perimeter Stateless Routing (GPSR), Adaptive Face Routing (AFR) and Greedy Other Adaptive Face Routing (GOAFR) [21].

\section{Yao-Gabriel Graph with Smart Boundaries}

In order to develop a technique that produced a network topology that met the objectives that have been set out and that adhered to the identified requirements and conformed to the choices made, it was decided to create a graph algorithm that is a hybrid of different proximity graph algorithms. The algorithm is a mixture of the Gabriel graph algorithm and the Yao graph algorithm, with the use of smart region boundaries. The algorithm is referred to as the Smart Boundary Yao Gabriel Graph (SBYaoGG). The topology is generated by first computing the Gabriel graph from the Unit Disk Graph (UDG) at maximum transmitter power and then computing the Yao graph on the reduced topology to produce the final topology.

By computing the Gabriel graph from the UDG some of the requirements for the final topology are met:

- The graph produced is planar.

- The graph has a linear number of edges.

After computing the Yao graph from the Gabriel graph some other requirements for the final topology are met:

- The graph is connected. This is because both the Gabriel graph and the Yao graph are connected if the original graph is connected.

- The graph is a power spanner of the original UDG. This is because both the Yao graph and the Gabriel graph are power spanners.

In addition to this:

- The final graph is easily computable in a distributed and localized way. The algorithm uses node position information which can be obtained from neighbor nodes. The edges are then computed locally by using only the neighbor position information.

Therefore, all of the requirements for the topology control technique were met. This contributed to meeting the objectives of the final graph in that it is energy efficient and has low interference. A low physical node degree necessary for minimal interference and a low power stretch factor necessary 
for high energy efficiency are two opposing goals, as has been noted.

1) Pruning the Edges of the Gabriel Graph

The Gabriel graph computed on the UDG has its edges pruned by computing the Yao graph on the reduced topology. This, in effect, generates the previously developed YaoGabriel graph. In order to achieve low interference, it is desirable to reduce the node degree as much as possible whilst maintaining the power spanner properties of the Gabriel graph. The YaoGabriel graph can achieve this. However, further reduction in interference levels can be obtained by variable selection of the axes of the cones for each region of the Yao graph.

The procedure employed to reduce interference was as follows:

- Prune the edges of the Gabriel graph using the Yao graph.

- Use large regions in computing the Yao graph.

- Select the axes of cones for each region of the Yao graph using heuristics.

- Reduce the transmitter power of each node to the lowest level so that it allows it to reach its furthest neighbor in the final topology.

2) Determining the Region Boundaries of the Yao Graph

A heuristic that was used whilst forming the reduced topology graph was to align the axis of the first cone used in the Yao graph computation to the region where nodes are most densely deployed. This can be accomplished by obtaining the unit direction vectors of all the neighboring nodes and then calculating the average direction vector. The average direction vector is then used as the axis of the cone for the first Yao graph region. The neighbor direction vectors and the average direction vector are illustrated in Fig. 1.

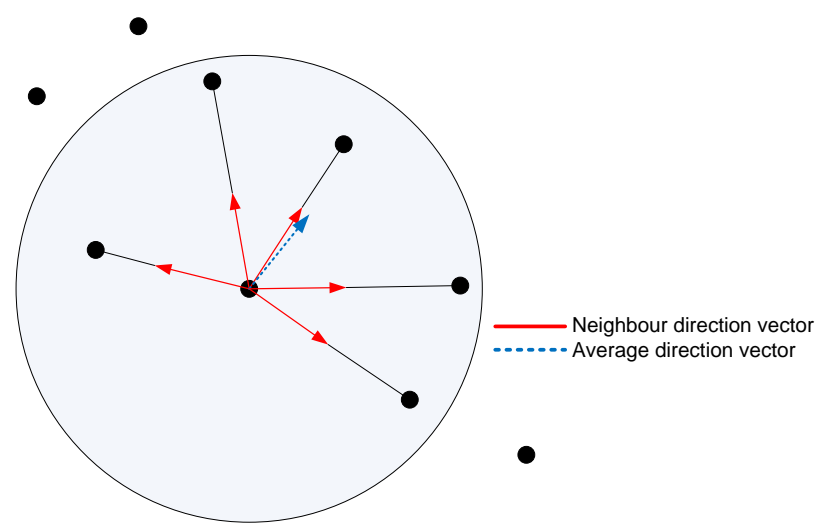

Fig. 1. Neighbor direction vectors and the average direction vector.

The cones of the Yao graph are as shown in Fig. 2. In the case of Figure $4-5 \alpha=120^{\circ}$, corresponding to a Yao graph with 3 cones. It can be seen that aligning the axis of one of the cones to the average direction vector, results in a cone where a high number of neighbor nodes fall into. It is possible that in certain arrangements all the neighbors will fall into this cone, which will mean that the number of edges calculated during topology control will be reduced.

An option was to use the neighbor centroid, for the following reason: Opposed to the average direction vector, the centroid has the drawback that neighbors that are further away will have coordinates that dominate over closer neighbor coordinates. The average of unit direction vectors overcomes this drawback. Selection of $\alpha \leq \frac{5 \pi}{6}$ is necessary to maintain connectivity [19]. The largest value of $\alpha=\frac{5 \pi}{6}$ was used in simulations when setting the boundaries of the Yao graph, in order to reduce the node degree as much as possible whilst maintaining connectivity.

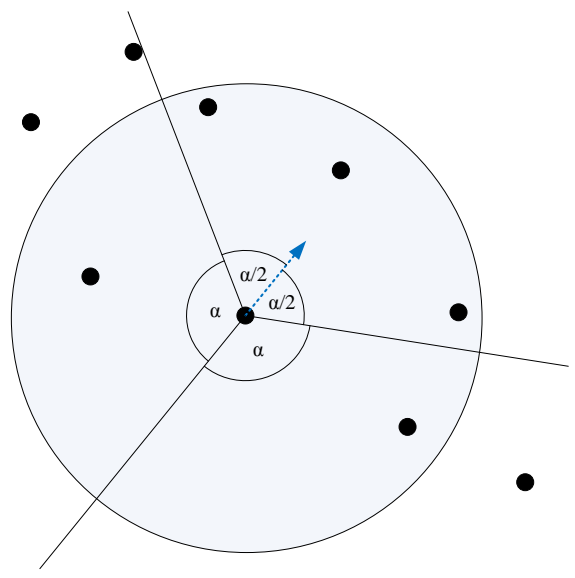

Fig. 2. Yao graph boundaries using an average direction vector.

3) Optimizations

Once the edges of the Gabriel graph are pruned, there will be some asymmetric edges. One optimization that was used was to make all the edges symmetric by adding the reverse edge of any asymmetric link where $u, v \in E$ but $v, u \notin E$. If a node $u$ has a link to a node $v$, but node $v$ does not have a link to node $u$, then node $v$ adds a link to node $u$. This reduces the power stretch factor of the final graph and ensures that there are no asymmetric links which can pose a burden to a MAC protocol.

4) Algorithm

The following algorithm describes how to construct the SBYaoGG, detailing the node in the network goes through.

Algorithm: Construction of SBYaoGG

1. The node discovers its neighbor nodes by broadcasting at maximum power.

2. The Gabriel graph is constructed locally.

3. The unit direction vectors of neighbor nodes in the Gabriel graph are computed.

4. The average direction vector is computed.

5. The axis of the cone of the first region to use in computing the Yao graph is set to correspond to the average direction vector.

6. The Yao graph is computed from the Gabriel graph, producing the reduced topology. 
The final step in obtaining the SBYaoGG is to optimize the reduced topology in order to ensure low interference and good power spanner properties. Two optimizations were made:

1. All edges are made symmetric by adding the reverse edge for any asymmetric link.

2. Transmitter power levels are set to the lowest level that will allow each node to reach all the nodes with which it has an edge.

After this the SBYaoGG is fully formed and can be used as input to a routing algorithm.

\section{RESULTS}

\section{A. Simulation Setup}

The SBYaoGG algorithm was run on several random networks in which nodes were distributed in a twodimensional unit square region according to a uniform random point process. 1000 samples were taken for each data point which gave results at a 95\% confidence level with an accuracy of close to $3 \%$. In addition to this, the algorithm was run on predetermined network deployments in order to not only evaluate the general performance of the algorithm, but also its situation-specific performance in networks that pose a challenge in some way in generating a topology with good performance. Custom simulator software was developed to run simulations. The original topology was a UDG formed at maximum communication range such that the graph is fully connected.

Different propagation models can be used to simulate various environment conditions, one thing that is common between the models is that the power required to communicate correctly between nodes increases as the Euclidean distance between them increases hence the use of the average edge length and distance stretch factor as measures of energy efficiency. Quality measures from section II.B were used.

\section{B. Predetermined Network Deployments}

The predetermined network deployments provide a quick way of comparing the topology control algorithm with other well-known algorithms and they help in visualizing how it works. Situations in which it is well suited are also made apparent.

\section{1) Double Ring Deployment}

In the double ring deployment the nodes are evenly distributed on the perimeters of two concentric circles with different radii, with one node located at the centre. This deployment can show topology control algorithms that are not degree-bounded and the energy efficiency of different algorithms. The topologies produced for the Delaunay graph, the S@GG, the SBYaoGG and the MST of the double ring network with 61 nodes, are shown in Fig. 3 (a) to (d) respectively and the performance metrics are show in Fig. 4.

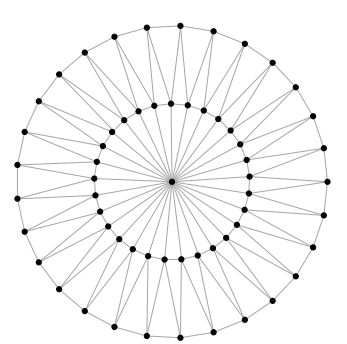

(a)

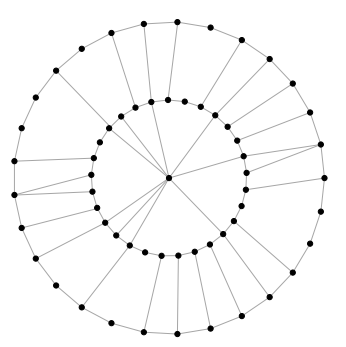

(c)

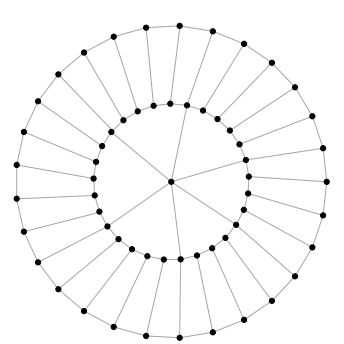

(b)

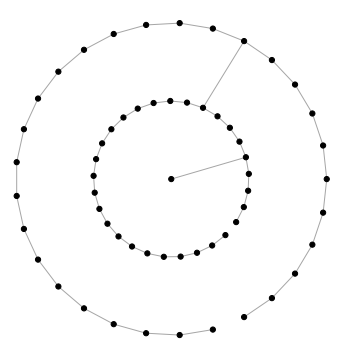

(d)
Fig. 3. Topology generated from the double ring network deployment for (a) DG, (b) S@GG, (c) SBYaoGG graph and (d) MST.

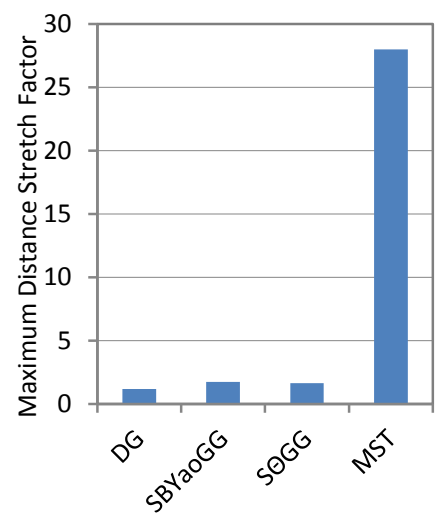

(a)

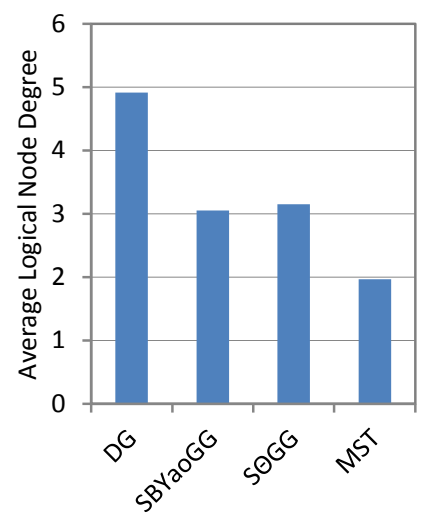

(c)

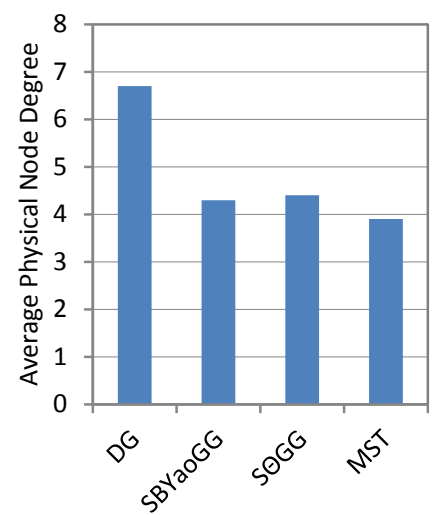

(b)

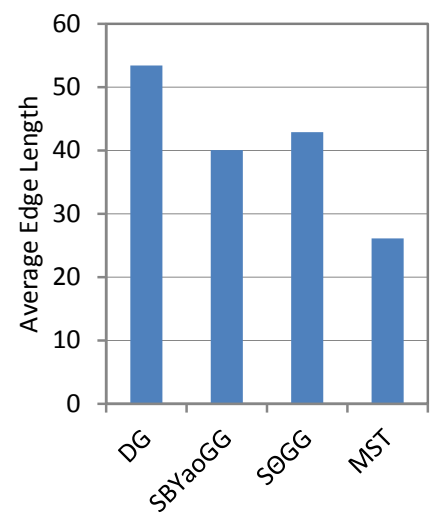

(d)
Fig. 4. Performance metrics obtained from double ring network deployment. 


\section{2) Exponential Node Chain Deployment}

The exponential node deployment is a one in which the distance between nodes increases exponentially [22]. This network can be used to compare the interference of different topology control algorithms. The topologies produced for the exponential node chain network with 10 nodes by the

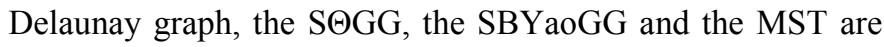
shown in Fig. 5 (a) to (d) respectively and the performance metrics are shown in Fig. 6.

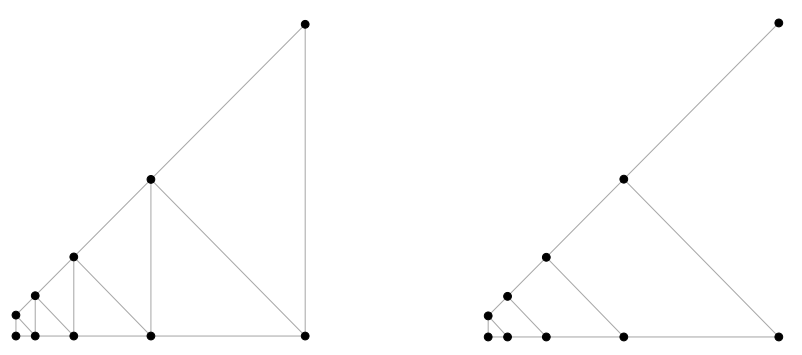

(a)

(b)

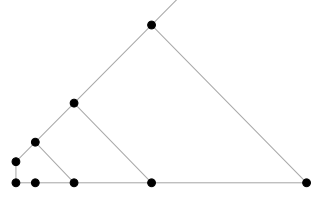

(c) (d)

Fig. 5. Topology generated from exponential node chain network deployment for (a) DG, (b) S@GG, (c) SBYaoGG graph and (d) MST.

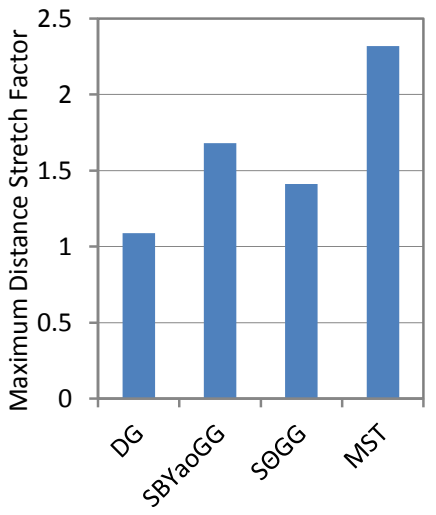

(a)

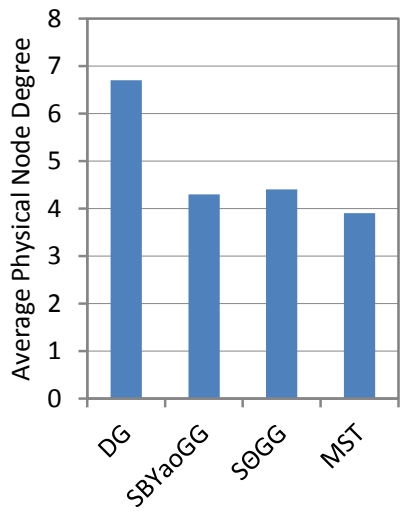

(b)

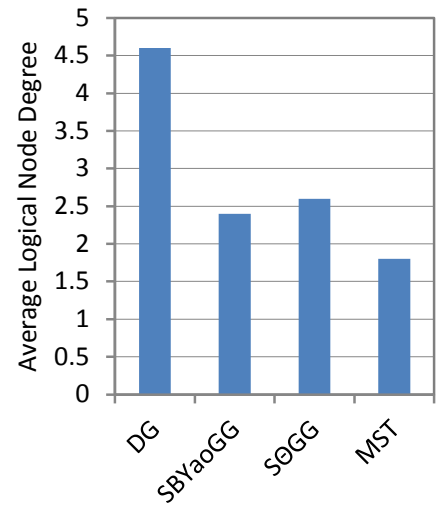

(c)

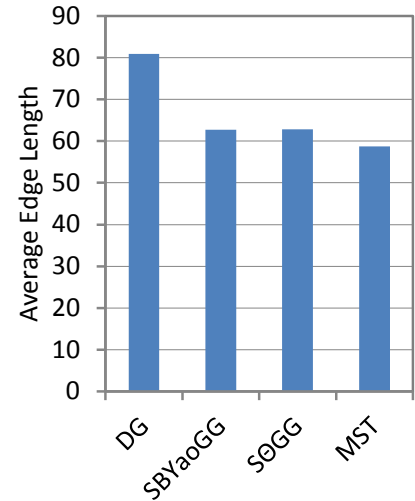

(d)
Fig. 6. Performance metrics obtained from exponential node chain network.

\section{3) Uniform Random Network Deployment}

In order to characterize the performance of a topology control algorithm that encompasses all sorts of network deployments it is useful to evaluate the algorithms based on their performance in several uniform random networks. Topologies generated by different topology control algorithms, including the SBYaoGG for the same uniform random network are shown in Fig. 7. The results obtained for several uniform random networks of different sizes are discussed in the next section.

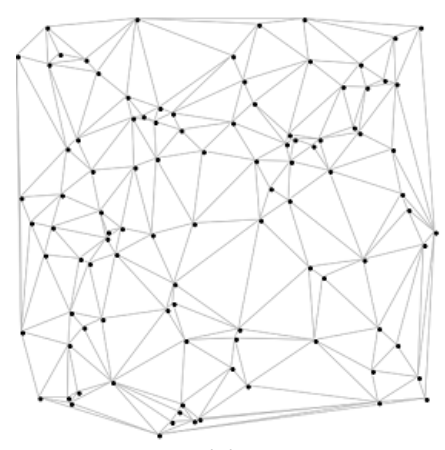

(a)

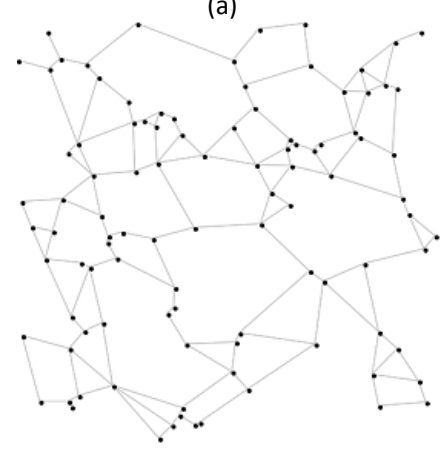

(c)

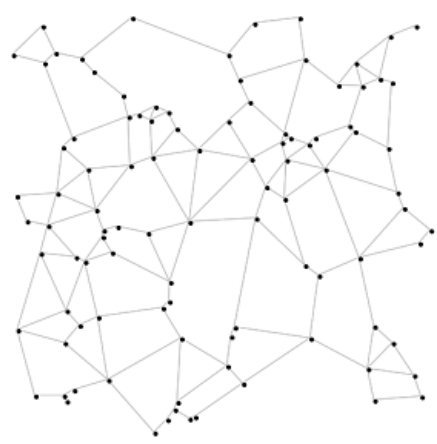

(b)

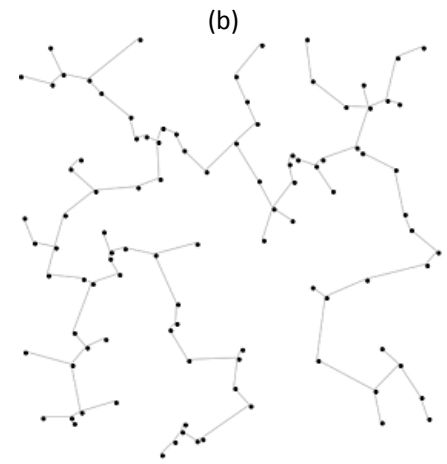

(d)
Fig. 7. Topology generated from a uniform random network deployment for (a) DG, (b) S@GG, (c) SBYaoGG graph and (d) MST. 


\section{Comparison with Other Algorithms}

In this section, the SBYaoGG will be compared to other topology control algorithms. The algorithms will be compared according to distance stretch factor, logical node degree, physical node degree, and edge length.

1) Distance Stretch factor

The maximum distance stretch factor shows energy efficiency in terms of end to end multi-hop communication from source to sink and not from hop to hop, which is represented by the average edge length. The maximum distance stretch factors of the SBYaoGG compared with the Delaunay graph, the Gabriel graph and the RNG are shown in Fig. 8. The Delaunay graph is shown to be the most energy efficient, followed by the Gabriel graph, the SBYaoGG and then the RNG.

It is worth noting that the MST produces the optimal energy efficient broadcast tree but does not optimize end to end communications between different nodes and hence performs very badly in this regard and is not useful when comparing distance stretch factors.

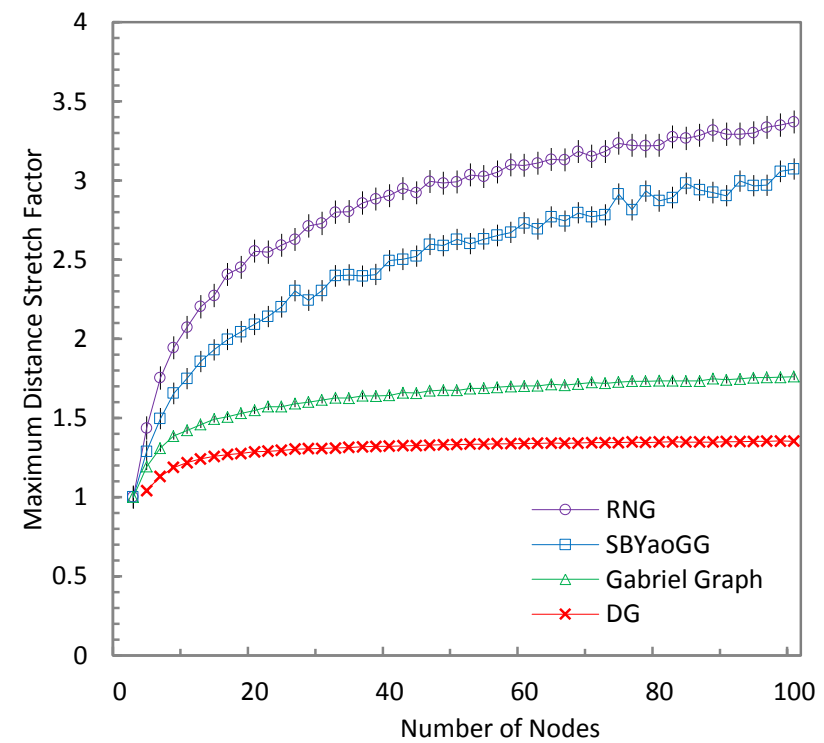

Fig. 8. Maximum distance stretch factor of different graphs.

\section{2) Logical Node Degree}

The average logical node degree shoes the number of neighbors a node will have and gives an indication of how big its routing table will be. The average logical node degree of the SBYaoGG compared with the Delaunay graph, the Gabriel graph, the RNG and the MST are shown Fig. 9. As can be expected, the MST has the lowest node degree, followed by the RNG while the Delaunay graph has the highest node degree, followed by the Gabriel graph. The SBYaoGG lies in between the RNG and the Gabriel graph and its node degree is lower than that of the Gabriel graph since it is a sub-graph of the Gabriel graph.

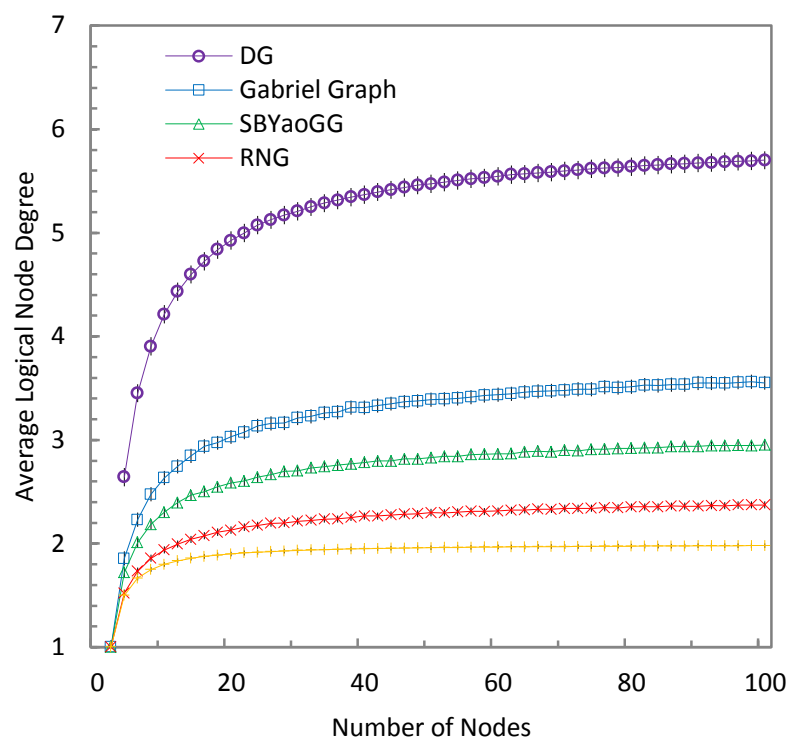

Fig. 9. Average logical node degree of different graphs.

3) Physical Node Degree

The average physical node degree show the number of nodes affected by transmissions from a single node and is a measure of interference and spatial reuse. The average physical node degree of the SBYaoGG compared with the Delaunay graph, the Gabriel graph, the RNG and the MST are shown in Fig. 10. The results are similar to those for the logical node degree, the MST will have the least interference and the SBYaoGG lies between the RNG and the Gabriel Graph.

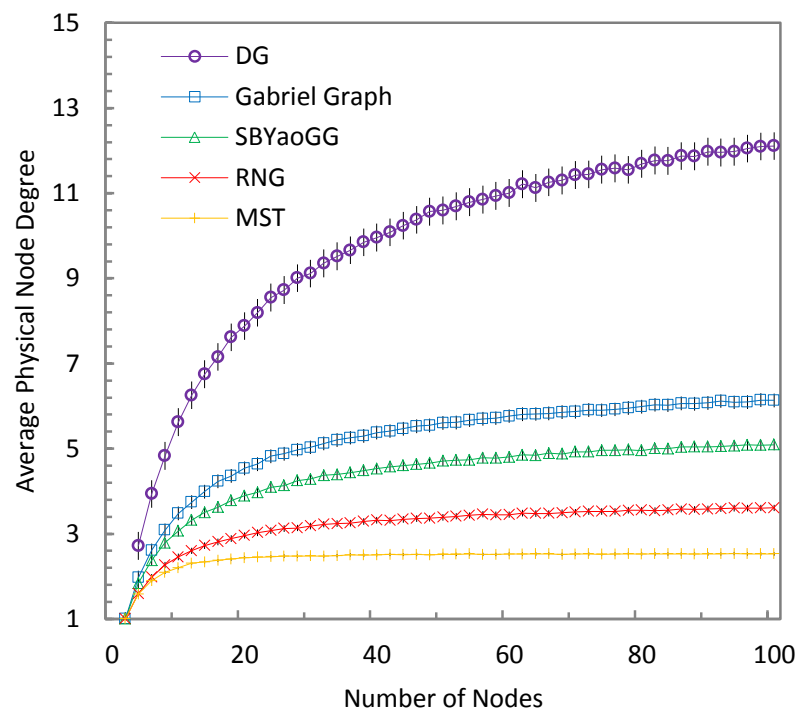

Fig. 10. Average physical node degree of different graphs. 


\section{4) Edge Length}

The average edge length shows energy efficiency in terms of hop to hop communication and is an indicator of individual node lifetime. The average edge length of the SBYaoGG, compared with the Delaunay graph, the Gabriel graph, the RNG and the MST are shown Fig. 11. The order of the results is the same as the results for the physical and logical node degree. This is because the MST is a sub-graph of the RNG, which is a sub-graph of the Gabriel graph, which is in turn a sub graph of the Delaunay graph. Also, the SBYaoGG is a sub-graph of the Gabriel graph.

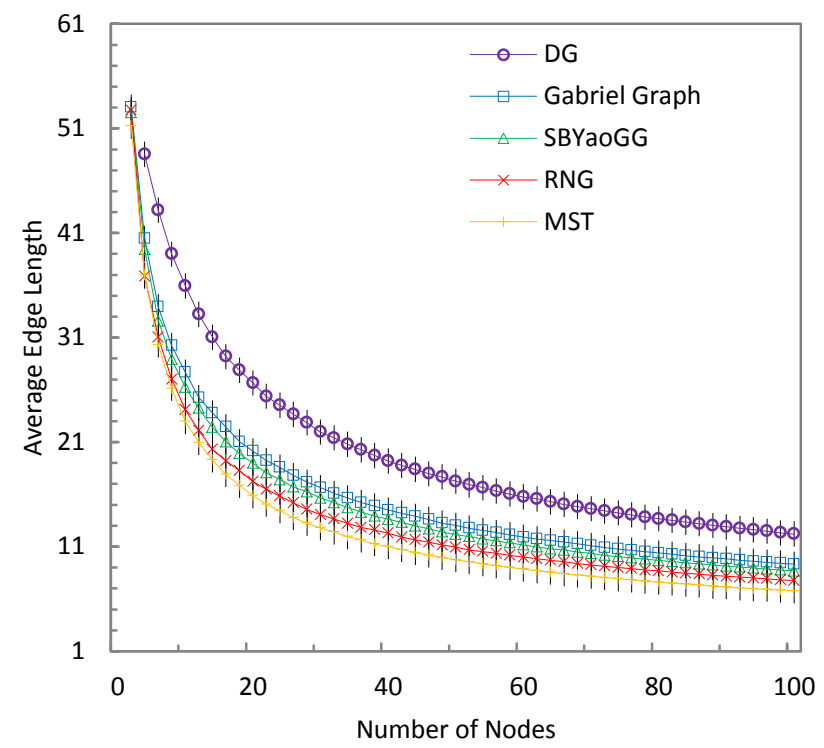

Fig. 11. Average edge length of different graphs.

\section{CONCLUSION}

In this research a topology control technique for energy efficient and low interference in wireless sensor networks was developed in the form of the SBYaoGG algorithm. The SBYaoGG algorithm was evaluated using a simulation program and was shown to perform favorably.

The SBYaoGG algorithm is a variation of the standard Yao Gabriel graph in which the boundaries of the regions of the Yao graph depend on the distribution of neighbors around a node. The average unit direction vector of the neighbors of a node is used as the axis of the first cone of the Yao graph. The SBYaoGG looks to develop as sparse a topology as possible with good power spanner properties. This is achieved by computing a Gabriel Graph from the original Unit Disk Graph and then computing the Yao Graph on the Gabriel Graph using smart region boundaries.

There are some heuristics that are used in order to produce as sparse a topology as possible whilst meeting the set out objective of good power efficiency. As few regions as possible were used to maintain connectivity of the graph when computing the Yao graph. Optimization steps are performed once the basic SBYaoGG is produced. The first step is to ensure that all links in the graph are symmetric by adding the reverse edge of all asymmetric links. This helps to ensure good energy efficiency by keeping the power spanning ratio down. The second optimization step is to set the transmitter power of each node to the minimum power that is necessary to reach the furthest neighbor with which it has a link in the topology controlled graph.

The performance of the SBYaoGG algorithm was evaluated using several performance metrics and compared with that of other topology control algorithms. The metrics that were chosen were those that measure energy efficiency, interference and the routing efficiency that will be a result of using the topology controlled graph as input to a routing protocol.

\section{REFERENCES}

[1] I. F. Akyildiz, W. Su, Y. Sankarasubramaniam, and E. Cayirci, "A survey on Sensor Networks", IEEE Communications Magazine, vol. 40, no. 8, pp. 102-10, 2002.

[2] M. M. Shalaby, M. A. Abdelmoneum, and K. Saitou, "Design of Spring Coupling for High-Q High-Frequency MEMS Filters for Wireless Applications", IEEE Transactions on Industrial Electronics, vol. 56, no. 4, pp. 1022-1030, 2009.

[3] A. Willig, "Recent and Emerging Topics in Wireless Industrial Communications: A Selection", IEEE Transactions on Industrial Informatics, vol. 4, no. 2, pp. 102-122, 2008.

[4] K. S. Low, W. N. N. Win, and M. J. Er, "Wireless Sensor Networks for Industrial Environments", in Proc. Computational Intelligence for Modelling, Control and Automation, 2005 and International Conference on Intelligent Agents, Web Technologies and Internet Commerce, 2005, pp. $271-276$.

[5] S. -E. Yoo et al., "Guaranteeing Real-Time Services for Industrial Wireless Sensor Networks with IEEE 802.15.4", IEEE Transactions on Industrial Electronics, vol. 57, no. 11, pp. 3868-3876, 2010.

[6] V. C. Gungor and G. P. Hancke, "Industrial Wireless Sensor Networks: Challenges, Design Principles, and Technical Approaches", IEEE Transactions on Industrial Electronics, vol. 56, no. 10, pp. 4258-4265, 2009.

[7] V. C. Gungor and F. C. Lambert, "A Survey on Communication Networks for Electric System Automation", Computer Networks, vol. 50, no. 7, pp. 877-897, 2006.

[8] V. C. Gungor, B. Lu, and G. P. Hancke, "Opportunities and Challenges of Wireless Sensor Networks in Smart Grid", IEEE Transactions on Industrial Electronics, vol. 57, no. 10, pp. 3557-3564, 2010.

[9] I. F. Akyildiz, T. Melodia, and K. R. Chowdhury, "A Survey on Wireless Multimedia Sensor Networks", Computer Networks, vol. 51, no. 4, pp. 921-960, 2007.

[10] A. Koubâa, R. Severino, M. Alves, and E. Tovar, "Improving Quality-ofService in Wireless Sensor Networks by Mitigating Hidden-Node Collisions", IEEE Transactions on Industrial Informatics, vol. 5, no. 3, pp. 299-313, 2009.

[11] S. Adee, (2010, February) IEEE Spectrum. [Online]. http://spectrum.ieee.org/semiconductors/devices/wireless-sensors-that$\underline{\text { live-forever }}$

[12] H. Karl and A. Willig, Protocols and Architectures for Wireless Sensor Networks, 1st ed. West Sussex, England: John Wiley \& Sons, 2006.

[13] L. Lobello and E. Toscano, "An Adaptive Approach to Topology Management in Large and Dense Real-Time Wireless Sensor Networks", IEEE Transactions on Industrial Informatics, vol. 5, no. 3, pp. 314-324, 2009.

[14] P. Santi, "Topology Control in Wireless Ad Hoc and Sensor Networks", ACM Computing Surveys, vol. 37, no. 2, pp. 164-194, 2005.

[15] R. Rajaraman, "Topology Control and Routing in Ad hoc Networks: A Survey", ACM SIGACT News, vol. 33, no. 1, pp. 60-73, 2002.

[16] K. J. Supowit, "The Relative Neighborhood Graph, with an Application to Minimum Spanning Trees", Journal of the ACM, vol. 30, no. 3, pp. 428-448, 1983.

[17] A. C. C. Yao, "On Constructing Minimum Spanning Trees in kdimensional Spaces and Related Problems", SIAM Journal of 
Computing, vol. 11, no. 4, pp. 721-736, 1982.

[18] N. Li, J. C. Hou, and L. Sha, "Design and Analysis of an MST-Based Topology Control Algorithm", IEEE Transactions on Wireless Communications, vol. 4, no. 3, pp. 1195-1206, 2005.

[19] L. Li, J. Y. Halpern, P. Bahl, Y.-M. Wang, and R. Wattenhofer, "A Cone-Based Distributed Topology-Control Algorithm for Wireless Multi-Hop Networks", IEEE/ACM Transactions on Networking, vol. 13, no. 1, pp. 147-159, 2005.

[20] D. M. Blough, M. Leoncini, G. Resta, and P. Santi, "The K-Neigh Protocol for Symmetric Topology Control in Ad Hoc Networks", in Proceedings of the International Symposium on Mobile Ad Hoc Networking and Computing (MobiHoc), 2003, pp. 141-152.

[21] W. -Z., Li, X. -Y., Frieder, O., Wang, W. Z. Song, "Localized Topology Control for Unicast and Broadcast in Wireless Ad Hoc Networks ", IEEE Transactions on Parallel and Distributed Systems, vol. 17, no. 4, pp. 321-334, 2006.

[22] P. von Rickenbach, R. Wattenhofer, and A. Zollinger, "Algorithmic Models of Interference in Wireless Ad Hoc and Sensor Networks", IEEE/ACM Transactions on Networking, vol. 17, no. 1, pp. 172-185, 2009.

[23] P. Santi and D. M. Blough, "The Critical Transmitting Range for Connectivity in Sparse Wireless Ad Hoc Networks", IEEE Transactions on Mobile Computing, vol. 2, no. 1, pp. 25-39, 2003.

[24] T. J. Kwon and M. Gerla, "Clustering with Power Control", in Proceedings - IEEE Military Communications Conference MILCOM 2, 1999, pp. 1424-1428.

[25] C. -F. Chiasserini, I. Chlamtac, P. Nucci, and A. Monti, "An EnergyEfficient Method for Nodes Assignment in Cluster-Based Ad Hoc Networks", Wireless Networks, vol. 10, no. 3, pp. 223-231, 2004.

[26] V. Kawadia and P. R. Kumar, "Principles and Protocols for Power Control in Wireless Ad Hoc Networks", IEEE Journal on Selected Areas in Communications, vol. 23, no. 1, pp. 76-88, 2005.

[27] Y. Xu, J. Heidemann, and D. Estrin, "Geography-Informed Energy Conservation for Ad Hoc Routing", in Proceedings of the Annual International Conference on Mobile Computing and Networking (MOBICOM), 2001, pp. 70-84.

[28] A. Cerpa and D. Estrin, "ASCENT: Adaptive Self-Configuring sEnsor Networks Topologies", IEEE Transactions on Mobile Computing, vol. 3, no. 3, pp. 272-285, 2004.

[29] M. D. Penrose, "On k-connectivity for a geometric random graph", Random Structures and Algorithms, vol. 15, no. 2, pp. 145-164, 1999.

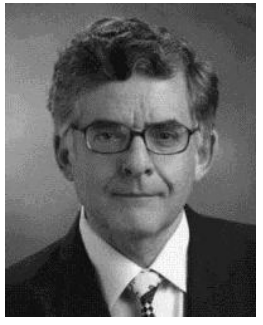

Gerhard P. Hancke (SM'00) received the B.Sc., B.Eng., and M.Eng. degrees from the University of Stellenbosch, Stellenbosch, South Africa, and the D.Eng. degree in 1983 from the University of Pretoria, Pretoria, South Africa.

$\mathrm{He}$ is currently a Professor and the Chair of the Computer Engineering Program and Research Group for Distributed Sensor Networks, University of Pretoria (a joint initiative between the Department of Electrical, Electronic and Computer Engineering and the Meraka Institute of the Council for Scientific and Industrial Research). His research interests primarily include distributed sensors and actuators networks.

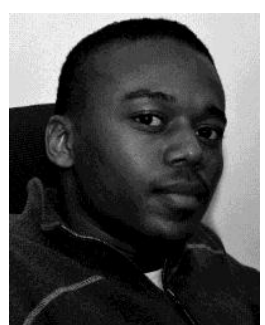

Tapiwa M. Chiwewe (S'09) received the B.Eng and M.Eng degrees in Computer Engineering from the University of Pretoria, Pretoria, South Africa, in 2006 and 2010 respectively.

$\mathrm{He}$ is currently working towards a Ph.D degree in Computer Engineering at the University of Pretoria. His research interests include wireless sensor networks and mobile computing. 